\title{
Characterization of the Bidirectional Vortex Using Particle Image Velocimetry
}

\author{
Brian A. Maicke and Joseph Majdalani \\ University of Tennessee Space Institute \\ United States of America
}

\section{Introduction}

The practical applications and theoretical difficulties associated with swirl-dominated flow fields have turned them into a source of constant scientific inquiry. Although some modern research efforts can be traced back to the piecewise formulation developed by Rankine (1858), an understanding of fundamental aspects of vortex dynamics has been demonstrated as far back as in ancient Greece (Vatistas, 2009). In fact, a multitude of naturally occurring phenomena such as hurricanes and tornadoes (Penner, 1972), as well as celestial events such as galactic jets are often modeled as vortex-dominated spiraling motions (Kirshner, 2004; Königl, 1986).

Confined vortex modeling involves small-scale helical motions that can be affected by the presence of viscous resistance. In naturally occurring vortices, an inviscid model is often sufficient as the natural length scales of the vortex substantially exceed the dimensions of the fluid regions influenced by viscous effects. However, in a confined vortex environment, the length scales are greatly reduced and therefore accounting for viscous effects becomes vital, especially near the axis of rotation.

A wealth of literature exists on confined vortex studies. For example, the efficiency of cyclone separators has been the focus of an investigation by ter Linden (1949). Bloor \& Ingham (1987) have also introduced an incompressible formulation for a conical separator in spherical coordinates. In addition to these practical applications, the confined vortex possesses important academic value. As far as stability of unidirectional vortices is concerned, Rusak et al. (1998) describe the evolution of a perturbed vortex in a pipe in an attempt to characterize axisymmetric vortex breakdown. This work is further extended by Rusak \& Lee (2004) to include compressible vortices. The intention of these studies is to not only characterize confined vortex breakdown, but to also extend the mechanisms entailed in generalized vortex breakdown to unconfined vortices.

\subsection{Experimental studies}

While only few analytical models have been proposed for describing the various swirl-dominated solutions of a confined vortex, there exists a significant body of literature that is devoted to experimental investigations. These studies can be roughly separated depending on the methods employed in their data collection: probes, Laser Doppler Velocimetry (LDV), and Particle Image Velocimetry (PIV). 


\subsubsection{Probes}

Within the context of cyclone separators, the experimental study by Smith (1962b) employs a glass tube filled with smoke particles to capture the general structure of a confined swirling flow. Smith also utilizes a special slender probe stretched across the diameter of the chamber to determine the magnitude and direction of the velocity in the cyclone. This setup allows the measurement of the axial and tangential components of the velocity. In this case, the radial velocity is assumed to be so small that it can be inferred from continuity. In a companion paper, Smith (1962a) combines analytical methods with experimental measurements to characterize the dynamics and possible instabilities that occur in a confined vortex.

In a later investigation into the behavior of cyclone combustors, Vatistas et al. (1986) conduct a similar experiment in which a prismatic pitot tube is used to capture the velocity and pressure maps within a cyclonic chamber. These researchers compare their findings to an experimentally correlated inviscid model and find what may be essentially viewed as a favorable agreement. Their study highlights a key realization in confined vortex modeling, namely, that swirl velocity variations in the axial direction are so small that they may be ignored (see Ogawa, 1984; Reydon \& Gauvin, 1981, among others). This simplification is common in the analytical modeling of vortices.

Furthermore, these studies provide early insights into the conditions arising in a confined vortex; however, some deficiencies must be noted. Even with proper calibration, the minimally intrusive probes can introduce disturbances into the flow, and these, in turn, can lead to potentially misleading results. This is especially important when investigating dynamic effects such as vortex instability.

\subsubsection{Laser Doppler Velocimetry}

Improvements in technology give rise to increasingly sophisticated experimental techniques that help to provide valuable information regarding confined swirl velocities without the intrusion invariably present with even the smallest probes. For instance, LDV minimizes flow disruptions by seeding the fluid domain with particles and then using a focused laser to scatter light off those particles. The interference patterns are then correlated to velocity measurements obtained in localized regions. Subsequently, the corresponding subvolumes are summed together to reconstruct the overall velocity profile of a given flow pattern.

Hoekstra et al. (1999) take an increasingly common approach of pairing a CFD solution with LDV measurements to validate their proposed turbulence models. Their experimental setup uses a back-scatter LDV to collect the axial and tangential velocity profiles in small volumes and these are then combined and correlated to provide an overall velocity profile. In this effort, however, the turbulent cross-correlation of the LDV measurements is found to be problematic because of the finite wall thickness of the cyclonic chamber which, in itself, can cause refraction and dissimilar levels of distortion based on the spatial location within the chamber. Without proper accounting for these optical disparities, a perfect correlation between the acquired signal and the flow profile will be difficult to realize. The quality of the seeding in the core region also proves to be an issue, as the natural motion in the cyclone tends to separate particles from the flow.

$\mathrm{Hu}$ et al. (2005) conduct a similar study for industrial-size cyclone separators. Whereas Hoekstra et al. (1999) focus on the separation section of the cyclone, $\mathrm{Hu}$ et al. (2005) 
consider the full geometry of the separator including the inlet, hoppers, and other supporting hardware. Moreover, their experimental investigation is accompanied by a turbulent computational solution. In addition to verifying turbulent vortex models, their study aims at improving the prediction of cyclone efficiency.

Along similar lines, an investigation into the turbulent kinetic energy of a confined vortex is reported in a forward scatter LDV study by Yan et al. (2000). In this work, data collected at a wide range of Reynolds numbers is used to validate their empirically derived solutions. These particular models rely on scaling laws to reduce the problem's dependence in each case to one or two key parameters and these tend to involve some combination of the inlet flow rate and the contraction ratio.

\subsubsection{Particle Image Velocimetry}

PIV is another minimally intrusive technique that will be discussed in the remainder of this chapter. Much like LDV, PIV employs particle seeding to collect velocity measurements. The primary difference between the two techniques stands in how the data is gathered. Whereas LDV relies on two focused beams to generate interference patterns, PIV uses optics to create a laser sheet that illuminates a plane in the chamber. High-speed cameras are then utilized to capture images of the illuminated particles at two closely spaced intervals such that a net velocity profile may be deduced from the cross-correlation of these images.

By way of comparison, both PIV and LDV methods are used by Sousa (2008) to determine the velocity field that accompanies vortex breakdown in a closed container. Sousa finds that accurate measurements may be acquired using either method; he also reports several challenges that may be associated with PIV techniques. The fully three-dimensional nature of the flow field can lead to a decrease in correlation accuracy as seed particles move normal to the light sheet. Sousa accounts for this factor by shortening the duration between laser pulses and by slightly thickening the laser sheet to increase the chances that the particles of interest will remain in the area of investigation.

In the spirit of improvement, Zhang \& Hugo (2006) use a stereoscopic PIV setup to investigate the vortex motion in a pipe. Stereoscopic PIV can be used to capture the fully three-dimensional flow field; however, it requires an additional high speed camera and more elaborate calibration skills to ensure that both cameras will target the same area. This obviously leads to an increase in post-experimental processing as the images from two cameras have to be analyzed for each exposure, effectively doubling the amount of data acquired. Finally, Zhang \& Hugo (2006) develop and implement an improved calibration technique to reduce the optical distortion caused by refraction through the fluid and the curved chamber wall.

\subsection{Classical solutions}

While a large body of literature may be reported on vortical motions, the segment devoted to analytical modeling remains much smaller in comparison. For this reason, a brief introduction to some of these classical models will follow.

In examining the classic models for describing swirl-dominated flowfields, it is helpful to further discriminate between solutions by specifying whether the attendant equations allow for unidirectional or multidirectional swirl. Unidirectional models are characterized 
by a one-directional axial velocity, whereas multidirectional motions exhibit a reverse flow character in which the axial velocity switches polarity. In practical applications, multidirectional flows are reduced to a bidirectional profile in which the axial velocity reverses only once.

\subsubsection{Unidirectional models}

One of the earliest unidirectional models is provided by Rankine (1858). Accordingly, the swirl velocity is formulated as a simple piecewise solution that is radially dependent. A normalized representation of this model may be written as

$$
\frac{\bar{u}_{\theta}}{\left(\bar{u}_{\theta}\right)_{\max }}=\left\{\begin{array}{ll}
\frac{\bar{r}}{\delta_{c}} & \bar{r} \leq \delta_{c} \\
\frac{\delta_{c}}{\bar{r}} & r>\delta_{c}
\end{array} \quad \text { or } \quad u_{\theta}= \begin{cases}r & r \leq 1 \\
r^{-1} & r>1\end{cases}\right.
$$

where overbars denote dimensional quantities and the radius is referenced to $\delta_{c}$, the distance from the origin to the point at which $\bar{u}_{\theta}$ reaches its peak value, $\left(\bar{u}_{\theta}\right)_{\max }$. The profile consists of a forced core that exhibits solid body rotation. The outer vortex varies with the inverse of the radius, so the swirl velocity diminishes away from the axis of rotation. Rankine's model is often used as a baseline solution for comparison or initialization owing to its simplicity. Nonetheless, Eq. (1) is somewhat limited in that the optimal matching location, $\delta_{\mathcal{C}}$, cannot be specified a priori, but requires data for anchoring. Moreover, the model is not differentiable at the matching location, which may be undesirable in subsequent analysis.

The Lamb-Oseen vortex consists of another solution that incorporates a time-dependent decay of the vortex motion (Wendt, 2001). This makes the model particularly suitable for capturing the behavior of wing-tip vortices. The dimensional representation of its swirl velocity may be expressed as

$$
\bar{u}_{\theta}(\bar{r}, \bar{t})=\frac{\Gamma}{2 \pi \bar{r}}\left[1-\exp \left(-\frac{\bar{r}^{2}}{\delta^{2}}\right)\right]
$$

Here $\Gamma$ refers to the circulation and $\delta=2 \sqrt{v \bar{t}}$, to the characteristic radius which is dependent on time, $\bar{t}$, and the kinematic viscosity, $v$. Equation (2) starts as a potential vortex, behaving as $1 / \bar{r}$ away from the centerline before smoothly switching to a linear dependence on $\bar{r}$ in the forced vortex core evolving around $\bar{r}=0$. As time elapses, the vortex decays exponentially.

The Burgers-Rott vortex (Burgers, 1948) is similar in form to the Lamb-Oseen profile with two notable exceptions. First, rather than a time-dependent decay, the exponential function here is governed by the suction parameter, $A$. Secondly, the Burgers-Rott vortex possesses well-defined relations for the axial and radial velocities. It can be written as

$$
\begin{aligned}
& \bar{u}_{\theta}(\bar{r})=\frac{\Gamma}{2 \pi \bar{r}}\left[1-\exp \left(-\frac{\bar{r}^{2}}{\delta^{2}}\right)\right] \\
& \bar{u}_{r}(\bar{r})=-A \bar{r} ; \quad \bar{u}_{z}(\bar{z})=2 A \bar{z}
\end{aligned}
$$

where $\delta=\sqrt{2 v / A}$. The presence of an axial velocity and a suction parameter has proven useful in applications related to the modeling of thunderstorms. 


\subsubsection{Bidirectional solutions}

In what concerns bidirectional behavior, an unbounded bipolar solution to the Navier-Stokes equations is provided by Sullivan (1959). For what is essentially a two-celled vortex, Sullivan's inner region exhibits a descending axial velocity coupled with an outward radial motion. Conversely, the outer cell flows inwardly and up. The model itself can be written in an integral representation using

$$
\left\{\begin{array}{l}
\bar{u}_{\theta}(\bar{r})=\frac{\Gamma}{2 \pi \bar{r}} \frac{1}{H(\infty)} H\left(\frac{\bar{r}^{2}}{\delta^{2}}\right) ; \quad \delta=\sqrt{2 v / A} \\
H(x)=\int_{0}^{x} e^{f(t)} \mathrm{d} t ; \quad f(t)=-t+3 \int_{0}^{t}\left(1-e^{-y}\right) \frac{\mathrm{d} y}{y}
\end{array}\right.
$$

As before, $A$ denotes the suction strength and $v$, the kinematic viscosity. The corresponding axial and radial components may be expressed as

$$
\bar{u}_{z}(\bar{r}, \bar{z})=2 A \bar{z}\left[1-3 \exp \left(-\frac{\bar{r}^{2}}{\delta^{2}}\right)\right] ; \quad \bar{u}_{r}(\bar{r})=-A \bar{r}+\frac{6 v}{\bar{r}}\left[1-\exp \left(-\frac{\bar{r}^{2}}{\delta^{2}}\right)\right]
$$

It is the combination of the axial and radial velocities that makes the Sullivan vortex a suitable candidate for modeling tornadoes (Wu, 1986).

A comparison of the above-mentioned swirl velocities is presented in Fig. 1. In all cases, the equations are normalized such that their peak velocities occur at a dimensionless radius of one. This is accomplished by dividing the radius by $\delta_{c}$, which is the distance from the axis of rotation to the point where the maximum swirl velocity occurs. Traditionally, a diameter of $2 \delta_{c}$ may be used to define the thickness of the forced viscous core. While all of the models capture similar trends, a significant amount of variability in the profiles may be seen. The Rankine solution displays an abrupt change in behavior at the peak velocity. The remaining models exhibit smooth contours, with the Sullivan profile concentrating the swirl velocity to a narrower region than that of Burgers-Rott. Although not depicted, the Lamb-Oseen velocity becomes identical to that of Burgers-Rott especially that time dependence is not featured in this figure.

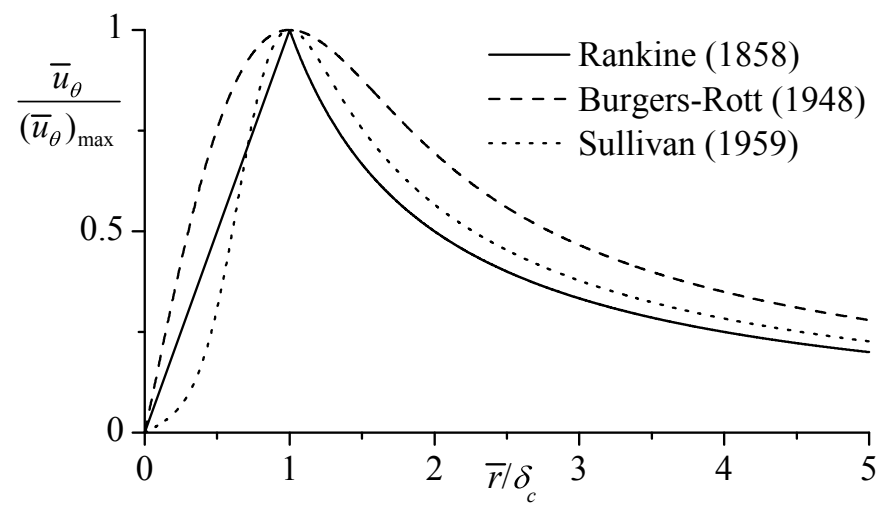

Fig. 1. A comparison of selected swirl velocity models, rescaled so that the peak velocity occurs at $r=1$. 
In work related to cyclone separators, a study by Bloor \& Ingham (1987) leads to one of the most frequently cited models. The resulting inviscid solution arises in the context of a conical cyclone (see Fig. 2). Bloor and Ingham solve the Bragg-Hawthorne equation in spherical coordinates to the extent of producing a stream function of the form

$$
\psi=\sigma R^{2}\left\{\left[\csc ^{2} \alpha+\ln \left(\tan \frac{\alpha}{2}\right)-\csc \alpha \cot \alpha\right] \sin ^{2} \phi-\sin ^{2} \phi \ln \left(\tan \frac{\phi}{2}\right)+\cos \phi-1\right\}
$$

Equation (6) translates into the following velocity components

$$
\begin{gathered}
u_{R}=2 \sigma R\left\{\left[\csc ^{2} \alpha+\ln \left(\tan \frac{\alpha}{2}\right)-\csc \alpha \cot \alpha\right] \cos \phi-\cos \phi \ln \left(\tan \frac{\phi}{2}\right)-1\right\} \\
u_{\phi}=\frac{2 \psi}{R^{2} \sin \phi} ; \quad u_{\theta}=\frac{1}{R \sin \phi} \sqrt{1-\frac{\bar{Q}_{i}^{2} \sigma \psi}{\pi^{2} a^{4} U^{2}}}
\end{gathered}
$$

Here $\bar{Q}_{i}$ denotes the volumetric flow rate through the cyclone, $U$ and $W$ stand for the average swirl and axial velocities at the entrance, $\alpha$ represents the taper angle of the cyclone, and $\sigma$ refers to the dimensionless swirl parameter described by

$$
\sigma=\frac{\pi a_{0}^{2} U^{2}}{\bar{Q}_{i} W}
$$

Here $a_{0}$ is the outer radius of the virtual inlet (see Fig. 2). Equations (6)- (9) constitute an improvement on previous work (Bloor \& Ingham, 1973), where use of the Polhausen technique leads to a solution that is insensitive to injection conditions. It should be noted that Eqs. (6)- (9) represent a corrected form of the Bloor-Ingham solution according to Barber \& Majdalani (2009).

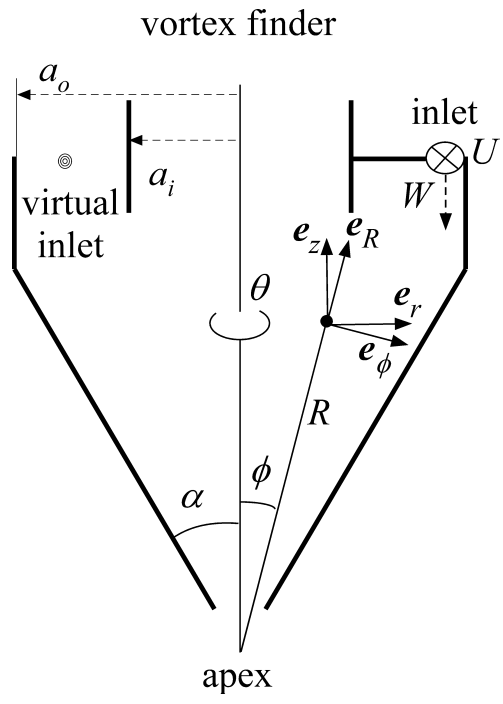

Fig. 2. Bloor-Ingham solution domain and geometry. 
Moving beyond the Bloor-Ingham approximation, Vyas \& Majdalani (2006) introduce a bidirectional model with a reversing axial character. Their complex-lamellar solution, which constitutes the basis for the upcoming analysis, seeks to describe the bulk gaseous motion in the Vortex Combustion Cold-Wall Chamber (VCCWC) developed by Chiaverini et al. (2002). In this vortex-driven engine, the swirling motion of the oxidizer insulates the sidewalls against thermal loading, thus leading to a substantial reduction in engine weight. The swirling motion also has a mitigating impact on pressure oscillations in the chamber as shown by Batterson \& Majdalani $(2011 a ; b)$. The mathematical character of this application is described in the following section.

The remainder of this chapter is focused on the synergistic combination of analytical and experimental methods in the context of confined vortex flows. First, two distinct analytical frameworks are devised with the intent of modeling the swirl velocity of a confined vortex. These models introduce control parameters that guide the construction of an experimental apparatus. A PIV setup is then used to capture the swirl velocity in the vortex chamber. Finally, the data is compared to the analytical frameworks in an effort to reconcile between theory and experiment.

\section{Analytical formulations}

The vortex chamber, shown in Fig. 3, is modeled as a right circular cylinder of radius $a$ and length $L$. The origin of the coordinate system is fixed at the center of the inert headwall, while the aft section is partially left open with a radius of $b$. The radial and axial coordinates are denoted by $\bar{r}$ and $\bar{z}$, respectively, while the characteristic geometric parameters are the outflow fraction $\beta=b / a$ and the aspect ratio $l=L / a$.

A single phase, non-reactive fluid is injected tangentially at $\bar{z}=L$. The fluid spirals up the outer annular region towards the headwall, thus forming an outer vortex (see Fig. 3). Once the fluid reaches the top of the chamber, it reverses axial direction and funnels out of the chamber as an inner vortex in the region $0<\bar{r}<b$. These two vortices are separated by a spinning, non-translating layer called the 'mantle.' This fluid interface materializes at $\bar{r}=b$. Mathematically, these conditions translate into the following boundary conditions:

$$
\begin{array}{lr}
\bar{r}=a, \bar{z}=L, \bar{u}_{\theta}=U & \begin{array}{r}
\text { tangential velocity at entry } \\
\bar{z}=0, \forall \bar{r}, \bar{u}_{z}=0
\end{array} \\
\bar{r}=0, \forall \bar{z}, \bar{u}_{r}=0 & \text { impervious headwall } \\
\bar{r}=a, \forall \bar{z}, \bar{u}_{r}=0 & \text { no flow across centerline } \\
2 \pi \int_{b}^{a} \bar{u}_{z}(\bar{r}, L) \bar{r} \mathrm{~d} \bar{r}=\bar{Q}_{i} & \text { impervious sidewall } \\
\bar{r}=0, \forall \bar{z}, \bar{u}_{\theta}=0 & \text { axial outflow matching tangential source } \\
\bar{r}=a, \bar{z}<L, \bar{u}_{\theta}=0 & \text { forced vortex center }
\end{array}
$$

The last two conditions are only used when accounting for viscous effects in the chamber. The $\bar{Q}_{i}$ term represents the inlet volumetric flow rate and $\bar{Q}_{0}$ is the outlet volumetric flow rate, calculated from the integral in (14). 


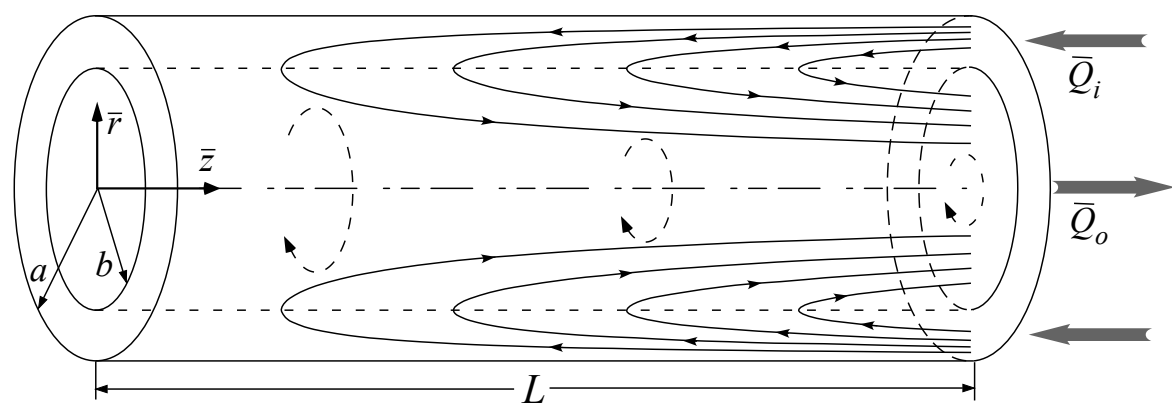

Fig. 3. Sketch of the bidirectional vortex chamber and corresponding coordinate system.

To facilitate comparisons to existing models, it is beneficial to use a non-dimensional form of the governing equations. This is achieved by setting

$$
\begin{gathered}
r=\frac{\bar{r}}{a} ; z=\frac{\bar{z}}{a} ; \delta=\frac{\bar{\delta}}{a} ; \nabla=a \bar{\nabla} ; \\
u_{r}=\frac{\bar{u}_{r}}{U} ; u_{\theta}=\frac{\bar{u}_{\theta}}{U} ; u_{z}=\frac{\bar{u}_{z}}{U} ; p=\frac{\bar{p}}{\rho U^{2}} ; Q_{i}=\frac{\bar{Q}_{i}}{U a^{2}}=\frac{A_{i}}{a^{2}}
\end{gathered}
$$

Here all spatial variables are normalized by the chamber radius and the velocities by the injection velocity. The variable $\delta$ represents the characteristic length scale used in the viscous analysis.

\subsection{Laminar core model}

The laminar core model is a solution to the Navier-Stokes equations using a stream function approximation. An inviscid base flow is obtained initially, followed by a boundary layer type correction at the centerline. In modeling confined vortices, it is common to assume that the flow is both axisymmetric and that the swirl velocity is axially invariant (see Leibovich, 1984). The equations for the inviscid flow, after applying these assumptions, reduce to:

$$
\begin{gathered}
\frac{1}{r} \frac{\partial\left(r u_{r}\right)}{\partial r}+\frac{\partial u_{z}}{\partial z}=0 \\
u_{r} \frac{\partial u_{r}}{\partial r}+u_{z} \frac{\partial u_{r}}{\partial z}-\frac{u_{\theta}^{2}}{r}=-\frac{1}{\rho} \frac{\partial p}{\partial r} \\
u_{r} \frac{\partial u_{\theta}}{\partial r}+\frac{u_{r} u_{\theta}}{r}=0 \\
u_{r} \frac{\partial u_{z}}{\partial r}+u_{z} \frac{\partial u_{z}}{\partial z}=-\frac{1}{\rho} \frac{\partial p}{\partial z}
\end{gathered}
$$

Equation (21) clearly shows the tangential velocity as fully decoupled from the radial and axial components such that it can be directly retrieved. Further application of the boundary condition from (10) gives

$$
u_{\theta}=\frac{1}{r}
$$


This free vortex form is common to many swirl-dominated inviscid flows. However, to realistically model the bidirectional vortex, viscous corrections will be required especially at the centerline.

With the swirl velocity successfully decoupled from the remaining two velocities, the Stokes stream function may be introduced using

$$
u_{r}=-\frac{1}{r} \frac{\partial \psi}{\partial z} ; u_{z}=\frac{1}{r} \frac{\partial \psi}{\partial r}
$$

Applying (24) and the vorticity transport equation (for additional detail see Vyas \& Majdalani, 2006) leads to the following relation in $\psi$, namely,

$$
\frac{\partial^{2} \psi}{\partial z^{2}}+\frac{\partial^{2} \psi}{\partial r^{2}}-\frac{1}{r} \frac{\partial \psi}{\partial r}+C^{2} r^{2} \psi=0
$$

Equation (25) can be solved via separation of variables. After applying the boundary conditions, the solution becomes

$$
\psi=\kappa z \sin \left(\pi r^{2}\right)
$$

where $\kappa$ is a constant determined from the mass conservation boundary condition in (14)

$$
\kappa=Q_{i} /(2 \pi l)
$$

The stream function provides the axial and radial velocity profiles which are key quantities in the calculation of the pressure. They may be expressed as

$$
u_{r}=-\kappa \sin \left(\pi r^{2}\right) / r ; \quad u_{z}=2 \pi \kappa z \cos \left(\pi r^{2}\right)
$$

With the choice of normalization being based on the average tangential speed at entry, the small size of $\kappa$ ensures that these two quantities remain of secondary importance relative to $u_{\theta}$.

The irrotational form in (23) accurately describes an inviscid vortex. However, for a confined vortex, some deficiencies arise, notably the singularity at the centerline where the velocity approaches infinity, and at the sidewall where slippage occurs. Bloor \& Ingham (1987) note that the unbounded behavior at the centerline appears to be a common characteristic of inviscid models to the extent of becoming archetypical (cf. Leibovich, 1984). To treat this core singularity, the second-order viscous terms in the $\theta$-momentum equation must be considered. Retention of these terms leads to

$$
u_{r} \frac{\partial u_{\theta}}{\partial r}+\frac{u_{r} u_{\theta}}{r}=\frac{1}{R e} \frac{\partial}{\partial r}\left[\frac{1}{r} \frac{\partial\left(r u_{\theta}\right)}{\partial r}\right] ; \operatorname{Re} \equiv \frac{\rho U a}{\mu}
$$

where Re represents the mean flow Reynolds number, $\mu$, the dynamic viscosity, and $\rho$, the density. In this particular case, $R e$ is of the order of $10^{5}$. Thus, a perturbation parameter may be formed by setting $\varepsilon \equiv 1 / R e$. For simplicity, the dimensionless angular momentum is consolidated into a single variable, $\xi \equiv r u_{\theta}$; this turns Eq. (29) into

$$
\varepsilon \frac{\mathrm{d}}{\mathrm{d} r}\left(\frac{1}{r} \frac{\mathrm{d} \xi}{\mathrm{d} r}\right)-\frac{u_{r}}{r} \frac{\mathrm{d} \xi}{\mathrm{d} r}=0 \quad \text { or } \quad \varepsilon \frac{\mathrm{d}}{\mathrm{d} r}\left(\frac{1}{r} \frac{\mathrm{d} \xi}{\mathrm{d} r}\right)+\frac{\kappa \sin \left(\pi r^{2}\right)}{r^{2}} \frac{\mathrm{d} \xi}{\mathrm{d} r}=0
$$


By converting the independent coordinate using $\eta \equiv \pi r^{2}$, Eq. (30) simplifies into

$$
\frac{\varepsilon}{\kappa} \frac{\mathrm{d}^{2} \xi}{\mathrm{d} \eta^{2}}+\frac{\sin \eta}{2 \eta} \frac{\mathrm{d} \xi}{\mathrm{d} \eta}=0
$$

In order to bring the swirl velocity to zero along the chamber axis, one must account for the rapid changes caused by the local emergence of viscous stresses (see Fig. 4). To do so, one may introduce the slowly varying scale, $s \equiv \eta / \delta(\varepsilon)$. The stretching transformation maps the region of non-uniformity about the centerline to an interval of order unity. Applying the transformation and linearizing the equation near the core where $s \approx 0$ yields

$$
\frac{\varepsilon}{\kappa \delta} \frac{\mathrm{d}^{2} \xi}{\mathrm{d} s^{2}}+\frac{1}{2}\left[1-\frac{\delta^{2} s^{2}}{3 !}+O\left(\delta^{2} s^{2}\right)\right] \frac{\mathrm{d} \xi}{\mathrm{d} s}=0
$$

The diffusive and convective terms strike a balance near the core. This occurs in (32) when $\delta \sim \varepsilon / \kappa$. Having identified the distinguished limit, the core boundary layer equation becomes

$$
\frac{\mathrm{d}^{2} \xi^{(i)}}{\mathrm{d} s^{2}}+\frac{1}{2} \frac{\mathrm{d} \xi^{(i)}}{\mathrm{d} s}=0
$$

where the superscript $(i)$ stands for the inner, near-core approximation. Using a standard perturbation series, successive viscous corrections can be determined to any desired order. For this chapter, a one-term inner solution is sought. Hence, by integrating (33) and insisting on a forced vortex near the core, one retrieves

$$
\xi^{(i)}=C_{0}\left[\exp \left(-\frac{1}{2} s-1\right)\right]
$$

The remaining constant may be determined through matching with the outer expansion. Using Prandtl's matching principle, the outer limit of the inner solution may be equated to the inner limit of the free vortex. This process results in a composite solution that is valid everywhere except in the close vicinity of the sidewall. After transforming back to the original coordinate, the composite inner solution collapses into

$$
\xi^{(c i)}=\bar{C}\left[1-\exp \left(-\frac{1}{4} V r^{2}\right)\right]
$$

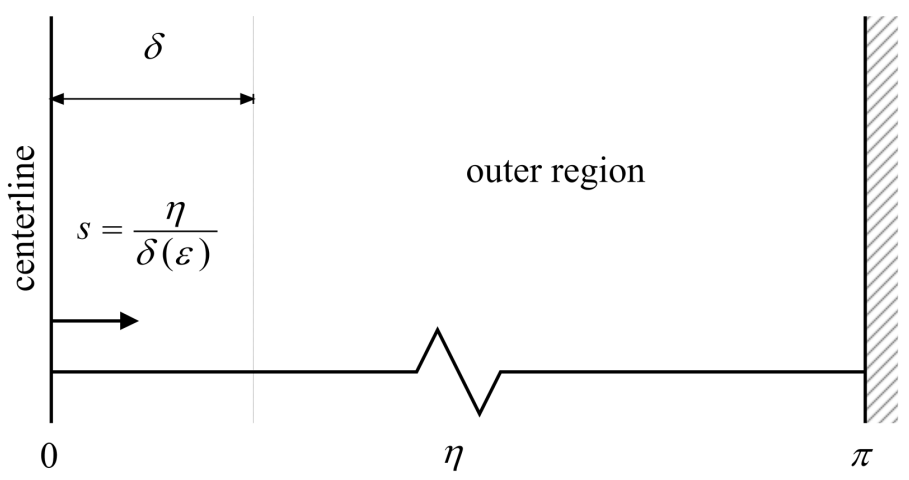

Fig. 4. Schematic of the dual end point boundary layers present in the confined vortex. 
where $V$ stands for the vortex Reynolds number,

$$
V \equiv \frac{1}{\varepsilon \sigma l}=\frac{\operatorname{Re}}{\sigma} \frac{a}{L}=\frac{\rho U A_{i}}{\mu L}=\frac{\dot{m}_{i}}{\mu L}
$$

For a complete physical description of $V$, the reader may consult with Majdalani \& Chiaverini (2009). The injection mass flow rate appears as $\dot{m}_{i}$ while the density and viscosity are given by $\rho$ and $\mu$. The outer constant $\bar{C}$ may be procured from the tangential injection condition. This implies

$$
\xi^{(c i)}(1)=1 \text { or } \bar{C} \simeq 1
$$

and so, when suitably combined, one has

$$
u_{\theta} \simeq \frac{1}{r}\left[1-\exp \left(-\frac{1}{4} V r^{2}\right)\right]
$$

Equation (38) provides a laminar representation of the swirl velocity in the bidirectional vortex chamber in which slip is permitted at the sidewall. To reconcile between this model and experimental data, additional steps are required as it will be shown below.

Before leaving this subject, it should be noted that a similar asymptotic analysis can be used to obtain the sidewall boundary layer correction. However, given the scope of this chapter, the attendant analysis is omitted. This is partly due to the fidelity of the PIV procedure and the data near the sidewall being too coarse to resolve the wall boundary layer. The additional complexity engendered by the sidewall correction is hence disregarded.

\subsection{Constant shear stress model}

The constant shear stress model provides a piecewise swirl velocity solution that may be used to model confined vortex motions. The basis for the model is that a free vortex of the $1 / r$ type develops away from the core of the vortex; in contrast, equilibrium is maintained near the core between the shear and pressure terms. This balance leads to a model that is equally valid for both laminar and turbulent conditions. It is important to note that the corresponding flow is not turbulent per se as there are no unsteady effects included, but rather a mean velocity profile that can be used to represent the bulk flow field in a turbulent regime.

The justification for this approach can be seen mathematically from the conservation of momentum, namely,

$$
(\boldsymbol{U} \cdot \nabla) \boldsymbol{U}=-\nabla p+\nabla \cdot \boldsymbol{\tau}
$$

At the centerline, the tangential velocity vanishes and this leaves the pressure and shear stress terms available to balance each other (Townsend, 1976). The flow under consideration for this work has a zero tangential pressure gradient; therefore, the shear stress in the tangential direction may be assumed to have a constant value. The equation for the dominant shear stress becomes

$$
\tau_{r \theta}=\epsilon\left[\frac{1}{r} \frac{\partial u}{\partial \theta}+r \frac{\partial}{\partial r}\left(\frac{v}{r}\right)\right]
$$

where $\epsilon$ is the same viscous parameter $1 / R e$. Because the flow is axisymmetric, the $\theta$ derivative is eliminated such that

$$
\epsilon\left(\frac{\partial v}{\partial r}-\frac{v}{r}\right)=C_{1}
$$


The traditional forced vortex model can be recovered by setting the constant equal to zero; nonetheless, the model examined here will retain the general constant. After integration, the inner swirl velocity becomes

$$
u_{\theta}^{(i)}=r\left[\frac{C_{1}}{\epsilon} \ln (r)+C_{2}\right]
$$

It may be interesting to note that each of the two undetermined constants, $C_{1}$ and $C_{2}$, has a clear physical meaning: while the first relates to the swirl strength of the velocity component generating the stress, the second corresponds to the swirl strength of a flow undergoing solid body rotation. The two undetermined constants can be manipulated to match the inner solution with the outer, free vortex expression at their intersection point. This is achieved by equating the velocity and its derivative to the outer vortex at a specific matching radius. However, since the matching radius is not known a priori, it must be carefully specified. For the moment, the matching point $X$ is left arbitrary. The equation to match the velocities at $X$ translates into

$$
X \frac{C_{1}}{\epsilon} \ln (X)+X C_{2}=\frac{1}{X}
$$

Equation (43) represents an effort to match the inner solution from (42) to the outer, free vortex solution. The same procedure can be used on the derivatives to provide

$$
\frac{C_{1}}{\epsilon}[1+\ln (X)]+C_{2}=\frac{1}{X^{2}}
$$

After solving (43) and (44) for $\left(C_{1}, C_{2}\right)$ and substituting back into (42), the result may be expressed as

$$
u_{\theta}= \begin{cases}\frac{r}{X^{2}}\left[1-\ln \left(\frac{r^{2}}{X^{2}}\right)\right] ; & r \leq X \\ \frac{1}{r} ; & r>X\end{cases}
$$

At this point in the analysis, the value of $X$ is still unknown although it produces a smooth function due to the matching conditions imposed through (43) and (44). The actual value of $X$ can be determined in a number of different ways, the foremost being a comparison to experimental data.

\section{Experimental setup}

With the analytical models for the confined vortex determined, it is possible to design a meaningful experiment through which collections of data can be obtained and compared directly to their theoretical predictions. Having identified the vortex Reynolds number, $V$, as the key parameter that governs the shape of the swirl velocity, any experimental setup must be able to incorporate a number of different values for $V$. When limited to a single working fluid, two ways exist for inducing variations in $V$ : changing the aspect ratio or the injection velocity. Rom (2006) uses this information to design a PIV experiment through which the character of the bidirectional vortex is carefully investigated.

To provide the necessary geometric flexibility, a modular chamber is used that allows for a variety of vortex Reynolds numbers. The chamber itself is made from a quartz cylinder and top plate. Four different chamber lengths provide a range of aspect ratios from 2.8 to 8.8. The bottom plate is acrylic and can be modified for a number of injection conditions. Additionally, 
the base of the chamber is retrofitted with a simple convergent nozzle with a throat diameter of $1.27 \mathrm{~cm}$ and an inlet radius of curvature of $0.635 \mathrm{~cm}$. Figure 5 showcases the modular chamber.

The working fluid for the PIV experiment is gaseous nitrogen. A number of configurable injectors are used to provide injection pressure drops that range from $10 \%$ to $30 \%$ of the chamber pressure. This, in turn, generates an increasing set of injection velocities. The nitrogen flow is seeded by a Corona Integrated Technologies Colt4 smoke generator. The process creates liquid particles of $0.2 \mu \mathrm{m}$ diameter.

The imaging system consists of a $250 \mathrm{~mJ} /$ pulse Nd:Yag laser to illuminate the seed particles. For this study, a typical pulse separation of $1 \mu$ s yields adequate resolution for the anticipated swirl velocities. The laser is focused through a series of adjustable optical devices to produce a sheet in the $r-\theta$ plane that can be positioned at three different axial locations. The particle images are captured by a LaVision $1280 \times 1024$ Flowmaster 3 camera at $1 \mu$ s increments which are spaced out over a thirty second run time. The images are then cross-correlated to deduce the swirl velocity at three axial locations in the chamber. A schematic of the setup is provided in Fig. 6.

The cross-correlation is carried out with LaVision DaVis 6.2 software. The two images, separated by $1 \mu \mathrm{s}$, are analyzed through deformed interrogation windows, initially $64 \times 64$ pixels in size. The windows decrease to $16 \times 16$ pixels during successive passes over the image. For each experimental configuration, ten sets of images are acquired and correlated to ten velocity fields.

In a related study by Rom et al. (2004), the PIV apparatus is supplemented with a modified end cap that is fitted with pressure taps. The taps are spaced at intervals of $15 \%$ of the radius with two additional taps at $r=0.9$ and 0.967 to capture the near wall behavior. The pressure measurements provide an additional avenue to verify the analytical approximations.

\subsection{Trial overview}

The modularity of the experimental apparatus will permit for a widely varying range of trials. For all trials, the radius of the chamber is kept fixed at $1.27 \mathrm{~cm}$. According to this arrangement, the smallest aspect ratio allows sampling at the midpoint of the chamber only. Table 1(a) provides the geometric variability of the test chamber.

In addition to the geometric variability, the injectors are configurable for three separate injection pressure drops. This is achieved by varying the available port area of the incoming fluid. All trials are conducted using eight equally spaced tangential injection ports. Details of the port construction are available in Table 1(b).

\section{Results}

After a successful trial, the ten pairs of image files are cross-correlated with DaVis 6.2 to produce a vector field for the swirl velocity, represented by a $128 \times 160$ matrix. Further data analysis is furnished via Matlab scripts which act upon the matrix exported by the DaVis software. The scripts average the ten raw velocity magnitudes at each axial location such that a radial profile of the swirl velocity may be reconstructed. The swirl velocity profile is an ideal candidate for comparison to the analytical models developed in Sec. 2. 


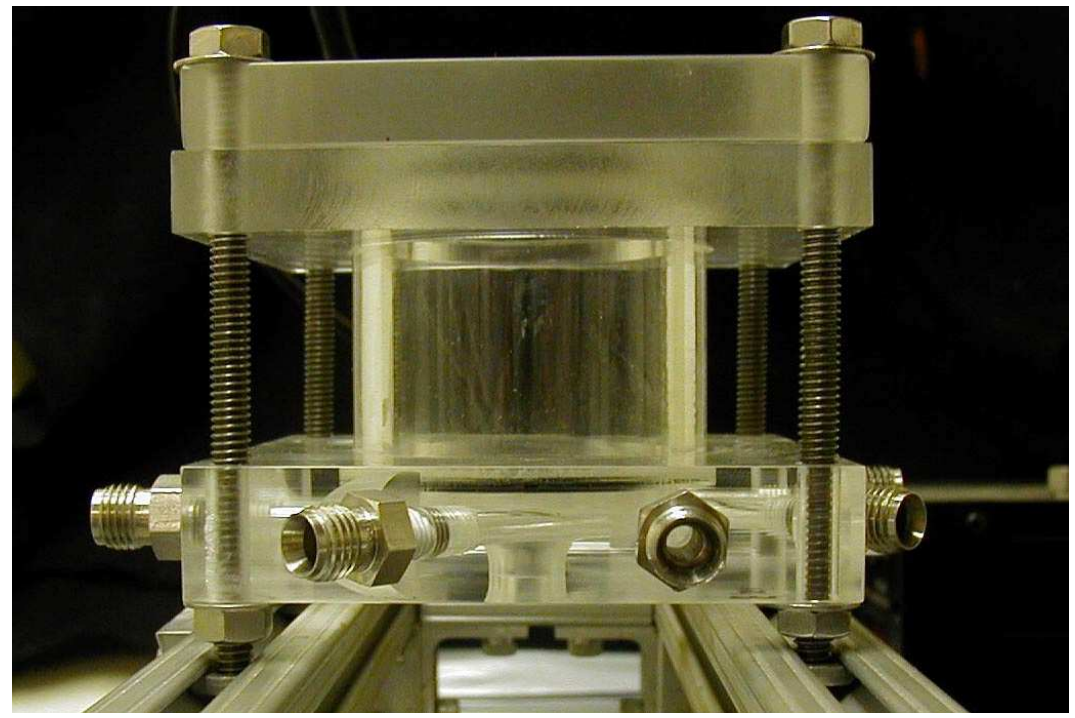

Fig. 5. Modular vortex chamber for the PIV experiment.

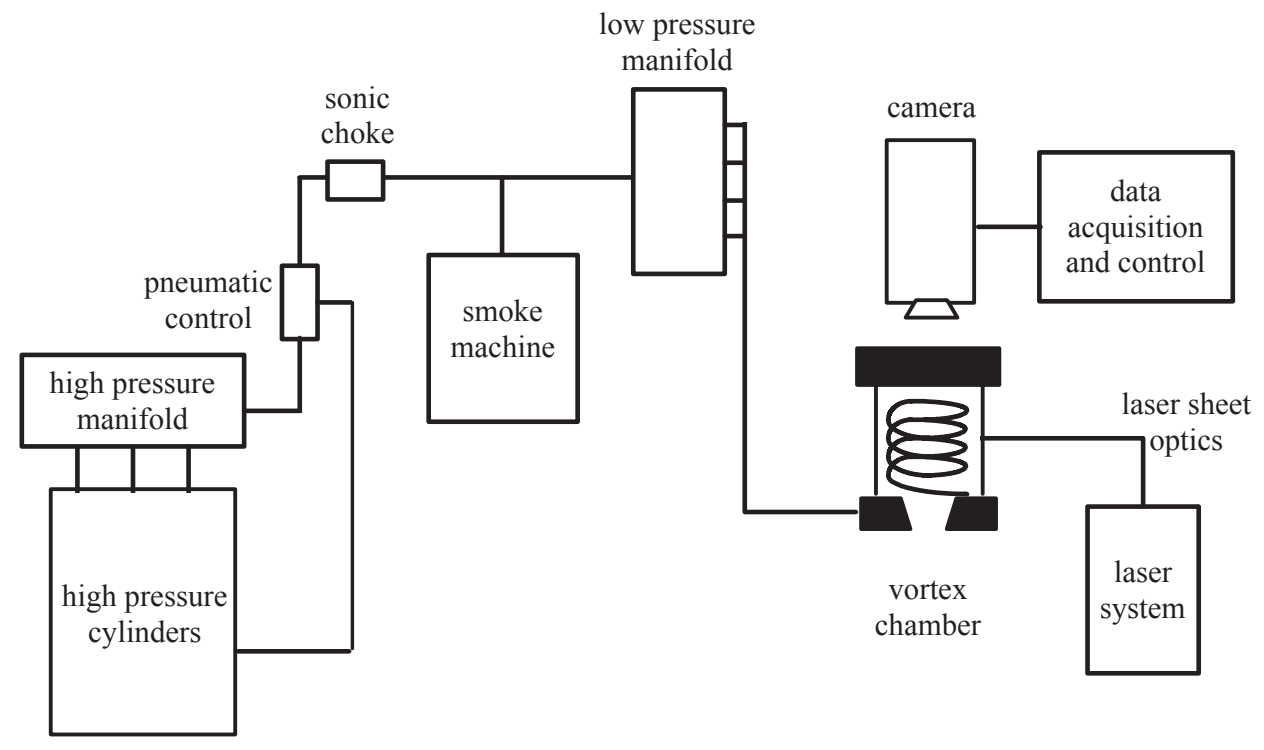

Fig. 6. Schematic of the PIV setup. 
(a) Geometric configurations

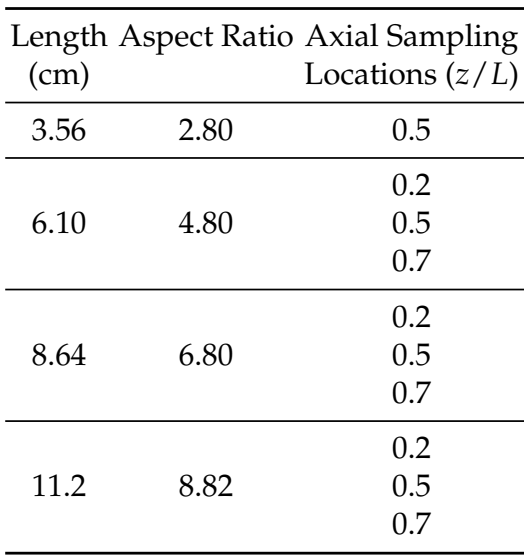

(b) Injector configurations

\begin{tabular}{ccc}
\hline $\begin{array}{c}\Delta p \\
\left(\% \text { of } p_{c}\right)\end{array}$ & $\begin{array}{c}\text { Port Dia. } \\
(\mathrm{cm})\end{array}$ & $\begin{array}{c}\text { Aggregate Injection } \\
\text { Area }\left(\mathrm{cm}^{2}\right)\end{array}$ \\
\hline 10 & 0.605 & 2.299 \\
20 & 0.500 & 1.571 \\
30 & 0.442 & 1.228 \\
\hline
\end{tabular}

Table 1. Available configurations for experimental trials.

\begin{tabular}{|c|c|c|c|c|c|c|}
\hline Parameter & Case 1 & Case 2 & Case 3 & Case 4 & Case 5 & Case 6 \\
\hline $\begin{array}{l}\text { Injector Pressure Drop, } \\
\qquad \Delta \bar{p}(\mathrm{kPa})\end{array}$ & 27.6 & 55.2 & 82.8 & 55.2 & 27.6 & 55.2 \\
\hline Aspect Ratio, $L$ & 2.4 & 2.4 & 4.4 & 3.4 & 4.4 & 4.4 \\
\hline $\begin{array}{l}\text { Average Injection Speed, } \\
U(\mathrm{~m} / \mathrm{s})\end{array}$ & 68.73 & 77.72 & 89.61 & 88.78 & 74.81 & 88.31 \\
\hline $\begin{array}{l}\text { Modified Swirl Number, } \\
\sigma=a^{2} / A_{i}\end{array}$ & 2.81 & 4.10 & 5.26 & 4.10 & 2.81 & 4.10 \\
\hline $\begin{array}{l}\text { Inflow Parameter, } \\
\qquad \kappa=1 /(2 \pi \sigma L)\end{array}$ & 0.0239 & 0.0164 & 0.0069 & 0.0114 & 0.0129 & 0.0088 \\
\hline $\begin{array}{l}\text { Vortex Reynolds Number, } \\
\qquad V=\dot{m}_{i} /\left(L_{0} \mu\right)\end{array}$ & 47150 & 36540 & 30160 & 29150 & 27650 & 22370 \\
\hline
\end{tabular}

Table 2. Operational parameters for the PIV experiments.

Six cases are chosen from the available trials for comparison with the laminar core and constant shear stress models. Data fidelity is the primary consideration in selection, as many of the other trials exhibit an increasing amount of scatter to the data, especially in the core region. Of the six cases considered, the first three are chosen to develop the empirically based correlations needed to match the data to the analytical approximation. The remaining three cases are held in reserve, to provide verification of the corrected models. The experimental parameters for these test cases are furnished in Table 2.

\subsection{Laminar core correlation}

In order to compare the analytical models to the experimental data, some additional effort is required. For the laminar core case, a simple least-squares regression will permit the vortex Reynolds number to be tuned to the experimental conditions. This correlation is necessary as the flow conditions in the experiment are beyond the range of validity of the laminar model. 


\begin{tabular}{ccccc}
\hline$V_{t}$ & $X_{0}$ & $X$ & $50 / \sqrt{V_{t}}$ & $\ell_{t}$ \\
\hline 47150 & 49.04 & 0.243 & 0.230 & 150.3 \\
36540 & 49.63 & 0.267 & 0.262 & 154.1 \\
30160 & 50.67 & 0.314 & 0.288 & 151.0 \\
\hline
\end{tabular}

Table 3. Least-squares parameters for laminar core and constant shear stress frameworks.

However, since the experimental vortex Reynolds number is also known, the test results can be linked to the analytical vortex Reynolds number through an empirically based viscosity ratio of the form $\ell_{t}=\mu / \mu_{t}$, with $\mu_{t}$ denoting a turbulent viscosity. In this manner, the vortex Reynolds number definition may be modified such that

$$
V=\frac{\rho U A_{i}}{\mu L}=\frac{\rho U A_{i}}{\mu_{t} \ell_{t} L}=\frac{V_{t}}{\ell_{t}}
$$

An average eddy viscosity may be calculated with this correlation that will properly match the laminar, analytical model to the high-speed experimental counterpart.

The standard least-squares regression scheme is used to determine the laminar vortex Reynolds number that best fits the model to the data. This value is then used to calculate the corresponding $\ell_{t}$ that will rectify the laminar and experimental vortex Reynolds numbers. The three trials provide 879 data points for the regression, producing an average $\ell_{t}=151.8$. Results of the least-squares regression runs for the individual trials are given in Table 3. Note that the three sets of experiments yield rather consistent values of $\ell_{t}=150,154$, and 151, respectively.

\subsection{Constant shear stress correlation}

The constant shear stress model requires a more elaborate analysis to achieve the same sort of comparison. First, the model itself consists of a piecewise function and the optimization parameter appears in both the solution and the boundary. To properly match the model to the experiment, a modified least-squares routine is developed using an iterative process that is capable of accounting for the moving boundary in the optimization.

\subsubsection{Piecewise least-squares regression}

The piecewise least-squares code contains several distinct components. The first element is a rewrite of the standard least-squares technique in a manner to incorporate the piecewise nature of the function into the derivative calculations. The function returns the optimized parameter, in this case the matching radius, $X$. The second function is simply a truncation function that adjusts the data set to reflect the new value of the optimization parameter. Finally, a control function loops over the data set, calling the least-squares function and comparing the new radius to the previous trial, $X$, until a satisfactory tolerance is reached, such as 0.0001 in this case. For the reader's convenience, a flow chart of the numerical procedure is provided in Fig. 7.

This iterative procedure is necessary because of the nature of this particular piecewise solution. For most piecewise solutions, a standard least-squares algorithm is sufficient. However, in this case, the optimization parameter coincides with the matching radius that determines the boundary between the inner and outer solutions. As a result, the optimization 
space changes every time that a new radius is calculated. The iterative approach continues to calculate new values of $X$ until the difference between successive radii falls below a user-specified tolerance. Since the data comprises a limited set of discrete points, the solution converges rapidly. The final radius is checked against neighboring values to ensure that the solution is in fact fully optimized.

\subsubsection{Viscous correlation}

Another fundamental issue associated with the shear stress framework is that the model is not written in terms of the vortex Reynolds number, $V$. The laminar core analysis highlights the importance of the vortex Reynolds number as it incorporates the geometry of the chamber, $l$, as well as the swirl dominated nature of the flow through the modified swirl number, $\sigma$. This parameter clearly determines the behavior of the swirl velocity near the core. To determine the vortex Reynolds number dependence, the constant shear stress model is matched to the laminar core solution such that both representations will return the same maximum velocity. Mathematically, this requires taking

$$
\left.\frac{\mathrm{d} u_{\theta}}{\mathrm{d} r}\right|_{r=r_{\max }}=0
$$

Solving Eq. (47) gives $r_{\max }=X / \sqrt{e}$. The same procedure applied to the laminar model yields

$$
r_{\max }=\sqrt{\frac{2}{V}\left[-1-2 \operatorname{pln}\left(-1,-\frac{1}{2} e^{-1 / 2}\right)\right]} \simeq \frac{2.242}{\sqrt{V}}
$$

With the maximum velocity locations known, these values may be substituted back into their respective expressions for velocity and then equated to produce

$$
\frac{2}{X e^{1 / 2}}=1-e^{\frac{1}{2}+\operatorname{pln}\left(-1,-\frac{1}{2} e^{-1 / 2}\right)} \sqrt{\frac{-V}{2\left[1+2 \operatorname{pln}\left(-1,-\frac{1}{2} e^{-1 / 2}\right)\right]}}
$$

Equation (49) may be solved for $X$ in terms of $V$ to obtain

$$
X=\frac{2}{\sqrt{e V}} \frac{\sqrt{-2\left[1+2 \operatorname{pln}\left(-1,-\frac{1}{2} e^{-\frac{1}{2}}\right)\right]}}{1-\exp \left[\frac{1}{2}+\operatorname{pln}\left(-1,-\frac{1}{2} e^{-\frac{1}{2}}\right)\right]} \simeq \frac{3.802}{\sqrt{V}}
$$

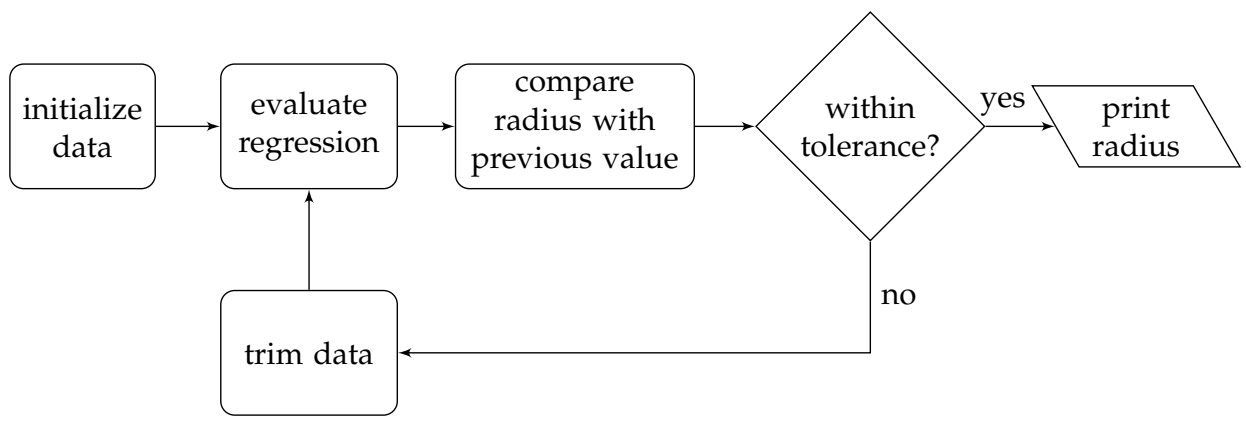

Fig. 7. Flowchart of the piecewise least-squares algorithm. 
This solution serves a twofold purpose. First, it demonstrates how the constant shear stress model can be matched to existing data as illustrated in Fig. 8. For additional matching paradigms the reader is directed to Maicke \& Majdalani (2009). Secondly, it provides an expected form of the relationship between $V_{t}$ and the matching radius. The iterative least-squares method uses the relationship

$$
X=\frac{X_{0}}{\sqrt{V_{t}}}
$$

The algorithm optimizes the constant $X_{0}$ to produce a matching radius for each trial that incorporates the experimental vortex Reynolds number. The calculated values are shown in Table 3 . Note that an average value of approximately 50 provides very good agreement with the experimental data.

\subsection{Graphical comparison}

Using $X_{0}=50$ and $\ell_{t}=151.8$, a comparison is drawn in Figs. 9 and 10 between theory and experiment. The measurements collected in each trial correspond to the data acquired at three axial locations in the chamber, specifically $z=0.2,0.5$, and 0.7 . The velocity plots in Fig. 9 display the collection of data used in the least-squares analysis. Consequently, good agreement is expected and achieved in all three figures. Figure 10 showcases the reserve data sets that were not employed in the regression algorithm, but rather saved to test the accuracy of the models at various vortex Reynolds numbers. As with the first three trials, the data in the outer, free vortex region is seen to be tightly grouped to the extent of faithfully following the shape predicted by both analytical models. Evidently, the agreement in the core region may be seen to be less appreciable. The additional scatter observed in the data is due to PIV limitations that will be discussed in Sec. 5. Nonetheless, the models do capture the general trends in the core. To accommodate the scatter, the fits for both theoretical models are weighted such that they provide an envelope in the core region, rather than a strict regression fit through the data. This weighting is chosen specifically because of the increased scatter in the core region that artificially lowers the predicted velocities.

It may be interesting to note the ability of the laminar core model with a turbulent eddy viscosity to duplicate the essential features of the flow. This behavior is consistent with

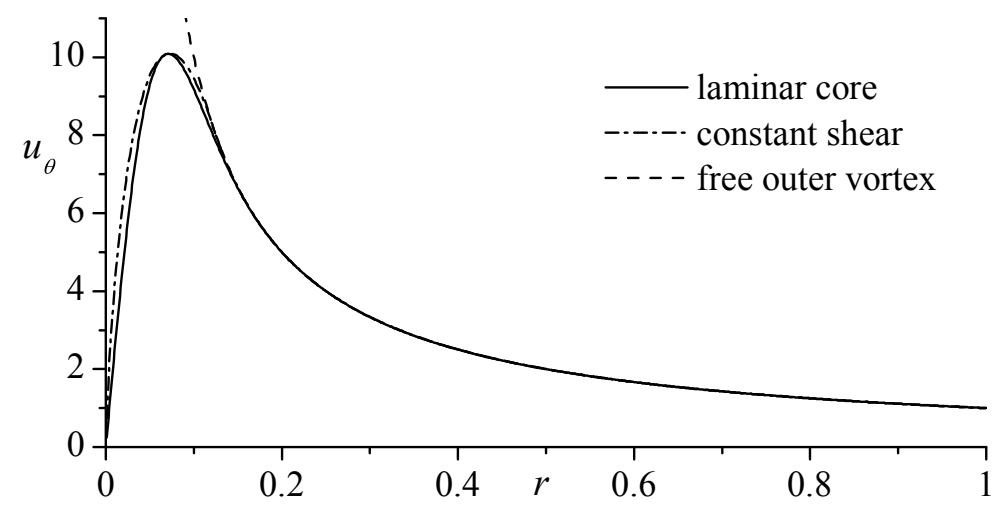

Fig. 8. Peak velocity matched models shown at $V=1000$. 


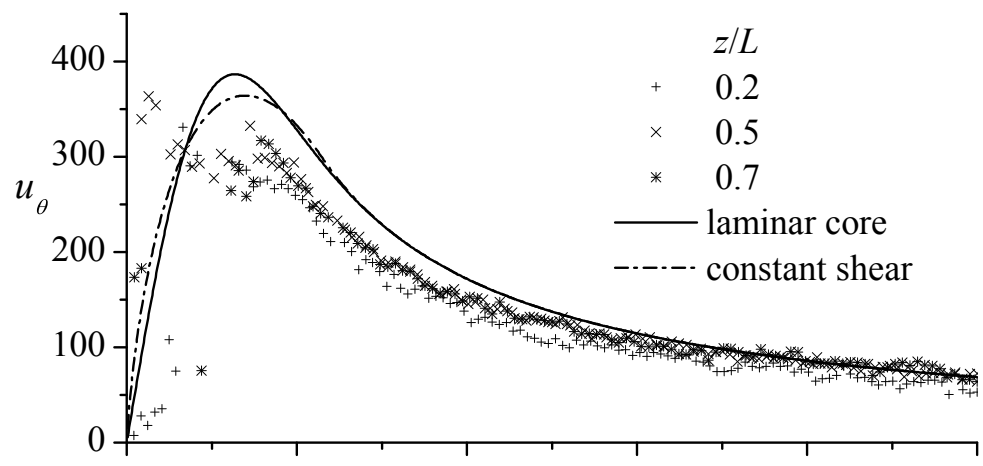

(a) $V=47150$

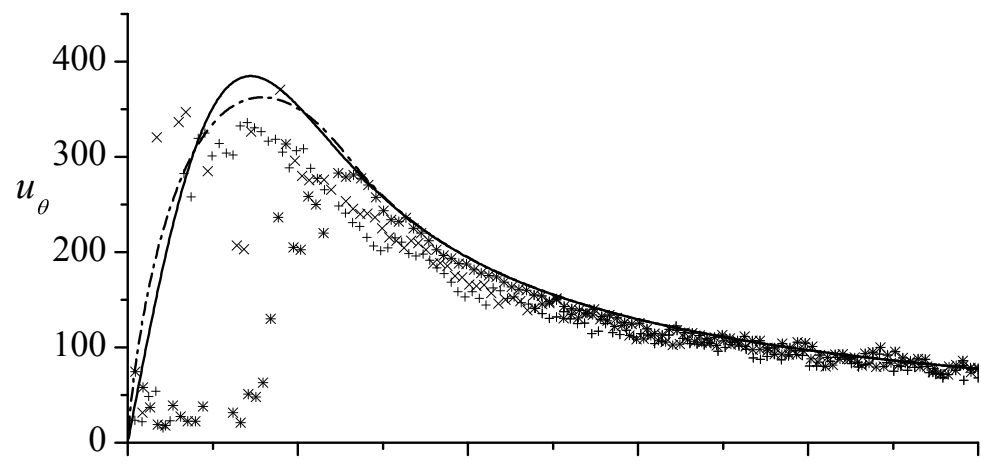

(b) $V=36540$

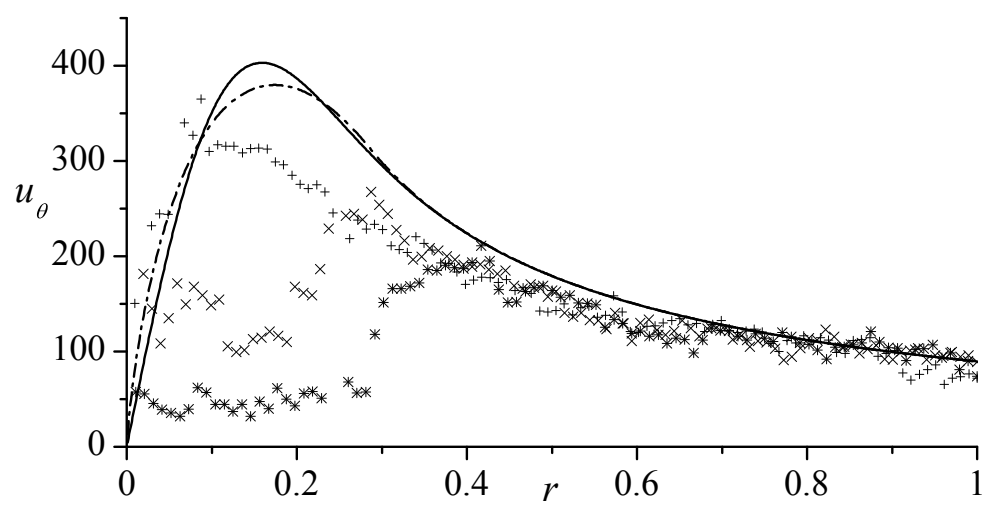

(c) $V=30160$

Fig. 9. Laminar and constant shear stress models compared to experimental data from Rom (2006) that is used in the least-squares regression. 


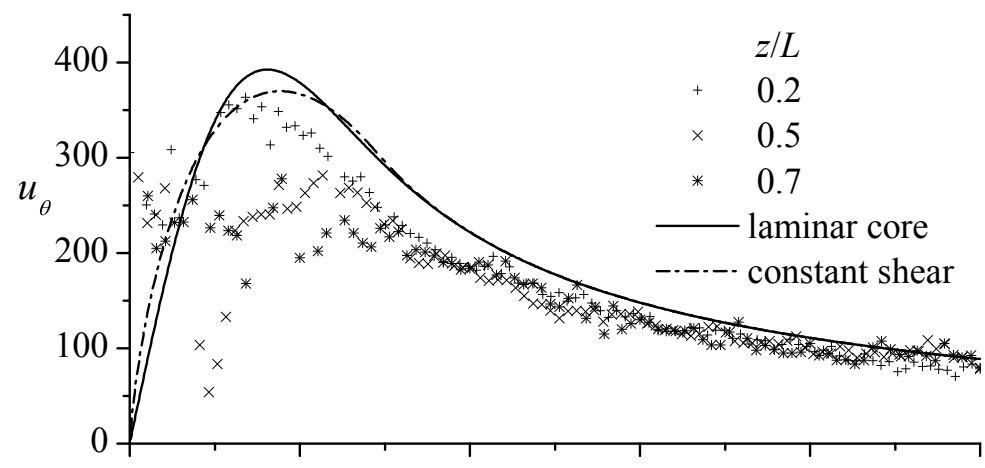

(a) $V=29150$

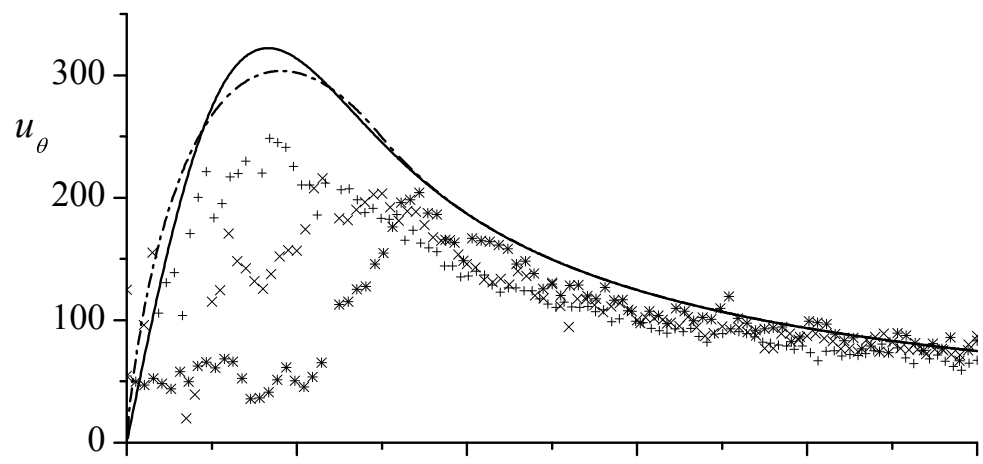

(b) $V=27650$

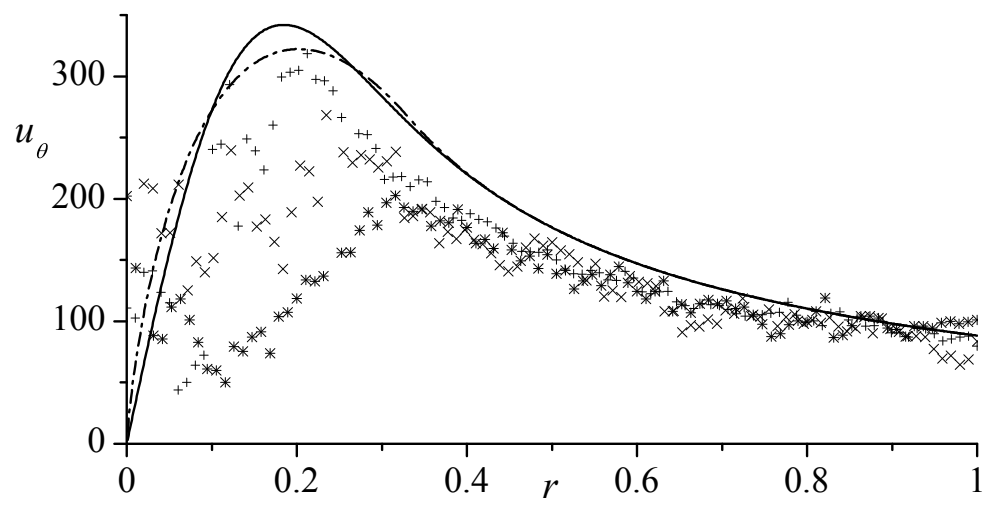

(c) $V=22370$

Fig. 10. Laminar and constant shear stress models compared to experimental data from Rom (2006) that is reserved for verification. 


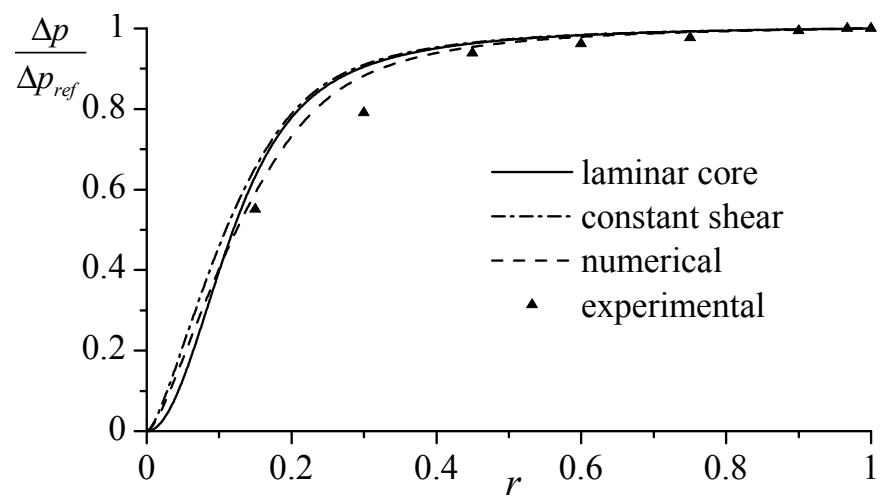

Fig. 11. Comparison of laminar core and constant shear stress models to experimental and numerical pressure data for $V_{t}=47150$.

the results of several experimental studies in which vortices are shown to exhibit an approximately constant angular velocity core with low turbulence levels to the extent of appearing as nearly laminar. With turbulent diffusion being restricted to an annular region about the laminar core, it is not surprising to see that the free vortex approximation in the outer domain continues to hold.

In addition to the swirl velocity distribution, the pressure measurements from Rom et al. (2004) may be used to verify the pressure predictions associated with the existing theoretical models. For the laminar core approximation, the equation of interest is

$$
\begin{aligned}
\Delta p= & -2 \pi^{2} \kappa^{2} z^{2} \\
& -\frac{1}{2} r^{-2}\left[\left(1-e^{-\frac{1}{4} V r^{2}}\right)^{2}+\kappa^{2} \sin ^{2}\left(\pi r^{2}\right)\right]+\frac{1}{4} V\left[\operatorname{Ei}\left(-\frac{1}{4} V r^{2}\right)-\operatorname{Ei}\left(-\frac{1}{2} V r^{2}\right)\right]
\end{aligned}
$$

where $\operatorname{Ei}(x)$ is the second exponential integral function. For the constant shear stress model, the piecewise relation for the pressure drop becomes

$$
\Delta p= \begin{cases}\frac{1}{2 \kappa^{4} r^{2}}\left(r^{4}\left\{5+\ln \left(\frac{r^{4}}{X^{4}}\right)\left[\ln \left(\frac{r}{X}\right)-2\right]\right\}-\kappa^{2} X^{4} \sin ^{2}\left(\pi r^{2}\right)\right) & r \leq X \\ +\frac{1}{2}-\frac{3}{X^{2}}-\kappa^{2}\left[4 \pi^{2} z+\frac{\sin ^{2}\left(\pi r^{2}\right)}{2 r^{2}}\right] ; & r>X \\ \frac{1}{2}-\frac{1}{2 r^{2}}\left[1+\kappa^{2} \sin ^{2}\left(\pi r^{2}\right)\right]-\kappa^{2}\left[4 \pi^{2} z+\frac{\sin ^{2}\left(\pi r^{2}\right)}{2 r^{2}}\right] ; & r \text {. }\end{cases}
$$

For more detail on the pressure relations, the reader is referred to Majdalani \& Chiaverini (2009) and Maicke \& Majdalani (2009).

Figure 11 depicts a normalized form of $\Delta p$ in the vortex chamber. Note that along with the experimental data, a numerical solution from Murray et al. (2004) is presented for the same conditions. All data sets are normalized to a reference value at the sidewall. The agreement displayed is encouraging especially near the wall region. Both analytical models exhibit 


\begin{tabular}{|c|c|c|c|c|c|c|c|c|c|}
\hline & \multicolumn{6}{|c|}{$\begin{array}{c}V_{t} \\
36540\end{array}$} & \multicolumn{3}{|c|}{30160} \\
\hline & $r_{c c}$ & $\sigma_{e}$ & $\Delta E_{t} \%$ & $r_{c c}$ & $\sigma_{e}$ & $\Delta E_{t} \%$ & $r_{c c}$ & $\sigma_{e}$ & $\Delta E_{t} \%$ \\
\hline laminar core & 0.887 & 0.592 & 4.91 & 0.962 & 0.276 & 1.47 & 0.870 & 0.391 & 3.45 \\
\hline constant shear & 0.900 & 0.558 & 4.36 & 0.968 & 0.253 & 1.23 & 0.880 & 0.376 & 3.19 \\
\hline
\end{tabular}

Table 4. Statistical parameters for the regression of the laminar and constant shear stress models.

a slight overprediction of the pressure, but follow the experimental trend admirably. The correlation of the theory with the numerical simulation appears to be excellent, with only slight deviations near the core region.

\subsection{Statistical verification}

To objectively compare the accuracy of the two models, several statistical parameters may be evaluated. By comparing correlation coefficients, $r_{c c}$, standard errors, $\sigma_{e}$, and total relative errors, $\Delta E_{t}$, the constant shear-based model seems to provide a slightly better fit to the data than the modified laminar distribution. The standard and total relative errors are calculated from

$$
\sigma_{e}=\frac{1}{\sqrt{n-1}} \sqrt{\sum_{i=1}^{n}\left[\hat{u}_{\theta}\left(r_{i}\right)-u_{\theta}\left(r_{i}\right)\right]^{2}}
$$

and

$$
\Delta E_{t}=\sum_{i=1}^{n}\left[\hat{u}_{\theta}\left(r_{i}\right)-u_{\theta}\left(r_{i}\right)\right]^{2} / \sum_{i=1}^{n} \hat{u}_{\theta}^{2}\left(r_{i}\right)
$$

where $n$ and $\hat{u}_{\theta}$ denote the number of data points and the measured velocity at $r_{i}$, the radius of the $i$ th data point. The standard error of the estimate quantifies the spread of data about the regression line, much like the standard deviation that measures the spread about a mean value. As shown in Table 4, the total relative error falls under 3.2, 1.3, and 4.4\% for the three cases associated with the constant shear stress approach. The corresponding experimental correlation coefficients are calculated at $0.88,0.97$ and 0.90 , respectively. When the modified laminar core technique is used, the relative errors slightly increase to $3.5,1.5$, and $5.0 \%$, with an equally minute reduction in $r_{c c}$.

The graphical and statistical verifications highlight the effectiveness of the theoretical approach that is pursued. The agreement between all cases, even those held in reserve, is particularly satisfying. However, additional experiments are necessary to both confirm the correlations developed here and to extend their usefulness to a wider variety of engine configurations and injection conditions.

\section{Challenges and limitations}

While the data provided by the PIV experiments proves invaluable in the verification of theoretical models, the technique is not without its challenges. In this section, the challenges and limitations of PIV applications to confined vortex flows are discussed.

In most cases, the PIV technique constitutes a powerful non-intrusive method for measuring velocities. However, as demonstrated in the previous sections, the vortical flow field can 
be difficult to capture in the region of maximum swirl, even with properly sized particles. In the core region, the particles exhibit increased drag as the swirl velocity reaches its peak. The particles also display a tendency to separate via centrifugal entrainment. Visually, this manifests itself as a reduction in the seed particles near the flow centerline. Furthermore, the core of the chamber happens to be the region of highest axial velocity; thus, the particles that are tagged in the first exposure may have traveled below the laser sheet by the time the second image is taken. This scenario can also lead to decreased confidence in the correlated data in the core region (see also Hu et al., 2005).

The verification process in this chapter is concentrated on a single aspect of the theoretical models, namely, the tangential velocity. To fully characterize the analytical approximations, the axial and radial velocities should be measured alongside the pressure. Practically, this cannot be accomplished in one trial with the current experimental configuration as the camera cannot track the fluid motion perpendicularly to the field of view. A stereoscopic PIV setup can overcome this deficiency albeit with increased cost in both equipment and procedural time as the pair of cameras require careful calibration in order to properly account for the velocities along the third axis. For this particular application, Laser Doppler Velocimetry may prove to be a better choice. In addition to providing velocity measurements in all three directions, a properly configured LDV experiment can produce data at more axial locations in a single setup. While not specific to PIV, acquiring the necessary pressure measurements may require yet another setup in which the clear headwall module can be replaced with a separate apparatus containing pressure taps.

Finally, the PIV approach cannot provide an accurate assessment of flow motion over the entire chamber. In the vicinity of the nozzle, accurate measurements are difficult to obtain as the fluid is restricted to a narrower cross-section; moreover, the character of the flow changes abruptly as it transitions from a swirl-dominated to a predominantly axial motion. In this region PIV is not sufficient to accurately resolve the velocity field; alternative techniques such as X-ray radiography may be required to more suitably investigate this region experimentally.

\section{Conclusion}

This chapter focuses on the use of PIV measurements in conjunction with two analytical models to describe the flow field in a bidirectional vortex chamber. Although the primary motivation for this work is to better understand the gas dynamics within a vortex-fired liquid rocket engine known as the Vortex Combustion Cold-Wall Chamber, the principal attributes associated with the resulting cyclonic motion have industrial, geophysical, meteorological, and astrophysical applications that fall beyond the propulsive dimension.

Using a cold-flow environment as a basis for this work, the findings indicate that both PIV and theoretical frameworks converge in predicting the presence of a forced vortex core region followed by a free, irrotational vortex tail that are characteristic of not only bidirectional vortices, but of unidirectional swirl-dominated flows as well. Moreover, despite the underlying restriction to non-reactive conditions, the essential features captured here seem to be somewhat representative of those reported in combustion chambers in which chemical reactions are prominent (Fang et al., 2004). It appears that the addition of high intensity swirl leads to a robust cyclonic motion that tends to retain its fundamental structure even in the presence of chemical kinetics. Nonetheless, more effort in modeling reactive and multiphase flows in cyclonic chambers will be critically important to further illuminate the particular 
mechanisms and parameters that control the performance of the VCCWC engine over a wide range of physical properties and geometric scales.

At this stage, the need to advance both computational and experimental capabilities in the modeling of reactive mixtures in swirl-dominated flows in general and cyclonic chambers in particular cannot be overrated. In what concerns the latter, it would be advantageous if a standard set of universal group parameters can be defined against which data acquired experimentally or numerically can be properly correlated. Thus guided by rigorous mathematical formulations that are derived from first principles, a basic set of dimensionless groupings such as the vortex Reynolds number, swirl parameter, inflow parameter, and chamber aspect ratio can be used consistently by various researchers while undertaking wide-range parametric studies. Presently, several different expressions of the swirl and vortex Reynolds numbers exist in the literature and this may be attributed to the dissimilar approaches used in defining them, be it rationalization, order-of-magnitude scaling, or conjecture. It would be strongly recommended that these are replaced by their corresponding forms that emerge naturally in analytical solutions that are obtained directly from first principles. The use of standard forms of these parameters will be essential before investing in new equipment and undertaking full-range laboratory or numerical experiments.

Novel investigations will be required to open up new lines of research inquiry and address several fundamental questions that remain unanswered. For example, it is still unclear what conditions will lead to the formation of a coherent cyclone, to vortex breakdown in a cyclonic chamber, or to the precession of the core vortex. It is also uncertain what techniques will be best suited to capture such behavior. The same may be said of acoustic and/or combustion instability of vortex-fired engines. Preliminary studies using the biglobal stability approach by Batterson \& Majdalani (2011a;b) show that stability is promoted with successive increases in swirl. Nonetheless, their study is limited to a narrow range of chamber aspect ratios and the hydrodynamic instability effects only represent one of the factors that must be accounted for in studying engine stability. The sidewall and headwall boundary layers, which were omitted in this chapter, pose another challenge that future examinations are hoped to overcome. Resolving the viscous stresses along the chamber wall will be essential not only to improve the velocity field description, but also to make any sort of formulation of a Nusselt number possible. This effort will have to be carried out using a three-pronged approach that leverages analytical, computational, and experimental techniques.

In hindsight, the availability of a laminar core flow solution to describe the bidirectional vortex has proven helpful in the design of a successful PIV experiment. The converse is also true given that the production of experimental data has been quintessential in refining the analytical approximations. The need to investigate the existence of more accurate, multi-dimensional, or higher-order helical solutions is evident. So far the analytical framework has provided several dimensionless parameters, including the vortex Reynolds number which is critical in controlling the flow behavior and providing guidance to minimize the number of experimental trials. The availability of a core shear stress model has also led to an iterative least-squares method that is specifically developed to enable comparisons between piecewise functions and experiments. To mitigate the bias in the unavoidable scatter in PIV data, the measurements have been carefully averaged and distilled in the process of calculating the turbulent eddy viscosity, $\mu_{t}$, and the corresponding viscosity ratio, $\ell_{t}=$ $\mu_{t} / \mu \simeq 152$, for the simulated vortex chamber. This effort epitomizes theory and experiment working hand-in-hand. In the absence of the PIV data, it would have been virtually impossible 
to predict the effective vortex Reynolds number, $V=V_{t} / \ell_{t}$, which will reduce the overshoot in the swirl velocity of the laminar core flow model. It is gratifying that the data generated from the PIV technique leads to a consistent agreement with the theoretical predictions despite the decreased fidelity in the core region.

Clearly, Particle Image Velocimetry seems to offer a vital resource in the quantification of confined vortex flows. Though not perfect, the technique captures the structure of the swirl velocity in the bidirectional vortex engine quite satisfactorily. By knowing the limitations of the method, specifically the likelihood of increased drag on particles near the peak flow velocity, it may be possible to refine the analytical models to the extent of better predicting their peak swirl velocities measured experimentally. Another aspect that must be brought into perspective is that, unlike the case of an unbounded vortex, the axial and radial velocities associated with confined vortices can have appreciable contributions in some regions of the flow domain. Then given that most studies to date emphasize the swirl velocity to the exclusion of the remaining components, more research is warranted to fully characterize the three-dimensional nature of the bidirectional vortex. This in turn will require a concerted effort between theoretical techniques and more advanced stereoscopic PIV or LDV procedures.

Because stereoscopic PIV relies on a rigorous procedure to capture the multidimensionality of complex flows, the addition of a second camera will be required to permit investigators to deduce particle velocities normal to the laser sheet. At the outset, all three components of velocity can be acquired at a given experimental section. With proper equipment, the light sheet can traverse the entire chamber to the extent of reproducing a comprehensive map of the vortex structure. In cases where the added expense of a stereoscopic apparatus proves impractical, a traditional PIV setup can still be employed to compile the necessary results, albeit with multiple trials.

An LDV technique may also be used in reconstructing the three-dimensional structure of the velocity field. In fact, Sousa (2008) relies on LDV measurements in his confined vortex study. However, a concern is raised in his investigation regarding the gains in spatial resolution that are offset by a substantially prolonged measurement time. In practice, each of these laser-based methods serves a different overall purpose. Whereas the PIV technique offers superb capabilities in capturing large scale velocity structures, LDV yields localized point measurements of fluid velocities. It can thus be seen that PIV may be the preferred method for visualizing large scale flow patterns such as those arising in cyclonic chambers.

In closing, it may be helpful to re-emphasize the tight balance between theory and experiment that stands behind this work. All too often, an investigation is steered towards one aspect of research to the detriment or neglect of the other. Without the theory to guide the proper design of experiments, more trials would have been necessitated to fully characterize the swirl velocity. Conversely, without the experimental values, the theoretical model could not have been refined and validated, nor could the turbulent eddy viscosity correlation been obtained. Pushing the borders of scientific inquiry certainly requires both theory and experiment to be pursued diligently and in unison.

\section{Acknowledgments}

This material is based on work supported partly by the National Science Foundation, and partly by the University of Tennessee Space Institute and the H. H. Arnold Chair of Excellence 
in Advanced Propulsion. The authors thank Dr. Martin J. Chiaverini of Orbital Technologies Corporation (ORBITEC) for providing the experimental data featured in this chapter.

\section{References}

Barber, T. A. \& Majdalani, J. (2009). Exact Eulerian solution of the conical bidirectional vortex, 45th AIAA/ASME/SAE/ASEE Joint Propulsion Conference and Exhibit, Denver, CO. AIAA Paper 2009-5306.

Batterson, J. \& Majdalani, J. (2011a). Biglobal instability of the bidirectional vortex. part 1: Formulation, 47th AIAA/ASME/SAE/ASEE Joint Propulsion Conference and Exhibit, San Diego, CA. AIAA Paper 2011-5648.

Batterson, J. \& Majdalani, J. (2011b). Biglobal instability of the bidirectional vortex. part 2: Complex lamellar and Beltramian motions, 47th AIAA/ASME/SAE/ASEE Joint Propulsion Conference and Exhibit, San Diego, CA. AIAA Paper 2011-5649.

Bloor, M. I. G. \& Ingham, D. B. (1973). Theoretical investigation of the flow in a conical hydrocyclone, Transactions of the Institution of Chemical Engineers 51(1): 36-41.

Bloor, M. I. G. \& Ingham, D. B. (1987). The flow in industrial cyclones, Journal of Fluid Mechanics 178: 507-519.

URL: $h$ ttp://dx.doi.org/10.1017/S0022112087001344

Burgers, J. M. (1948). A mathematical model illustrating the theory of turbulence, Advances in Applied Mechanics 1: 171-196.

Chiaverini, M. J., Malecki, M. J., Sauer, J. A. \& Knuth, W. H. (2002). Vortex combustion chamber development for future liquid rocket engine applications, 38th AIAA/ASME/SAE/ASEE Joint Propulsion Conference and Exhibit, Indianapolis, IN. AIAA Paper 2002-4149.

Fang, D., Majdalani, J. \& Chiaverini, M. J. (2004). Hot flow model of the vortex cold wall liquid rocket, 40th AIAA/ASME/SAE/ASEE Join Propulsion Conference and Exhibit. AIAA Paper 2004-3676.

Hoekstra, A. J., Derksen, J. J. \& van den Akker, H. E. A. (1999). An experimental and numerical study of turbulent swirling flow in gas cyclones, Chemical Engineering Science 54(13): 2055-2065.

URL: http://dx.doi.org/10.1016/S0009-2509(98)00373-X

Hu, L. Y., Zhou, L. X., Zhang, J. \& Shi, M. X. (2005). Studies of strongly swirling flows in the full space of a volute cyclone separator, AIChE Journal 51(3): 740-749.

URL: http://dx.doi.org/10.1002/aic.10354

Kirshner, R. P. (2004). The Extravagant Universe: Exploding Stars, Dark Energy, and the Accelerating Cosmos, Princeton University Press, Princeton, New Jersey.

Königl, A. (1986). Stellar and galactic jets: Theoretical issues, Canadian Journal of Physics 64: 362-368. URL: $h$ ttp://dx.doi.org/10.1139/p86-063

Leibovich, S. (1984). Vortex stability and breakdown: Survey and extension, AIAA Journal 22(9): 1192-1206.

URL: $h$ ttp://dx.doi.org/10.2514/3.8761

Maicke, B. A. \& Majdalani, J. (2009). A constant shear stress core flow model of the bidirectional vortex, Proceedings of the Royal Society, A 465: 915-935.

URL: $h t t p: / / d x$.doi.org/10.1098/rspa.2008.0342 
Majdalani, J. \& Chiaverini, M. J. (2009). On steady rotational cyclonic flows: The viscous bidirectional vortex, Physics of Fluids 21: 103603-15.

URL: $h t t p: / / d x$.doi.org/10.1063/1.3247186

Murray, A. L., Gudgen, A. J., Chiaverini, M. J., Sauer, J. A. \& Knuth, W. H. (2004). Numerical code development for simulating gel propellant combustion processes, 52nd JANNAF Propulsion Meeting, Las Vegas, NV. JANNAF Paper 2004-0115.

Ogawa, A. (1984). Estimation of the collection efficiencies of the three types of the cyclone dust collectors from the standpoint of the flow pattern in the cylindrical cyclone dust collectors, Bulletin of the JSME 27(223): 64-69.

Penner, S. S. (1972). Elementary considerations of the fluid mechanics of tornadoes and hurricanes, Acta Astronautica 17: 351-362.

Rankine, W. J. M. (1858). A Manual of Applied Mechanics, 9th edn, C. Griffin and Co., London, UK.

Reydon, R. F. \& Gauvin, W. H. (1981). Theoretical and experimental studies of confined vortex flow, The Canadian Journal of Chemical Engineering 59: 14-23.

URL: $h$ ttp://dx.doi.org/10.1002/cjce.5450590102

Rom, C. (2006). Flow field and near nozzle fuel spray characterizations for a cold flowing vortex engine, Master's thesis, University of Wisconsin-Madison, Madison, WI.

Rom, C. J., Anderson, M. H. \& Chiaverini, M. J. (2004). Cold flow analysis of a vortex chamber engine for gelled propellant combustor applications, 40th AIAA/ASME/SAE/ASEE Joint Propulsion Conference and Exhibit, Fort Lauderdale, FL. AIAA Paper 2004-3359.

Rusak, Z. \& Lee, J. H. (2004). On the stability of a compressible axisymmetric rotating flow in a pipe, Journal of Fluid Mechanics 501: 25-42. URL: $h t t p: / / d x$.doi.org/10.1017/S0022112003006906

Rusak, Z., Wang, S. \& Whiting, C. H. (1998). The evolution of a perturbed vortex in a pipe to axisymmetric vortex breakdown, Journal of Fluid Mechanics 366(1): 211-237.

Smith, J. L. (1962a). An analysis of the vortex flow in the cyclone separator, Journal of Basic Engineering 84(4): 609-618.

Smith, J. L. (1962b). An experimental study of the vortex in the cyclone separator, Journal of Basic Engineering 84(4): 602-608.

Sousa, J. M. M. (2008). Steady vortex breakdown in swirling flow inside a closed container: Numerical simulations, PIV and LDV measurements, Open Mechanical Engineering Journal 2: 69-74.

URL: http://dx.doi.org/10.2174/1874155X00802010069

Sullivan, R. D. (1959). A two-cell vortex solution of the Navier-Stokes equations, Journal of the Aerospace Sciences 26(11): 767-768.

ter Linden, A. J. (1949). Investigations into cyclone dust collectors, Proceedings of the Institution of Mechanical Engineers 160: 233-251.

Townsend, A. A. (1976). The Structure of Turbulent Shear Flow, Cambridge University Press.

Vatistas, G. H. (2009). Vorticies in Homer's Odysssey-A scientific approach, Science and Technology in Homeric Epics, History of Mechanism and Machine Science, Springer Netherlands, pp. 67-75.

URL: $h t t p: / / d x . d o i . o r g / 10.1007 / 978-1-4020-8784-4 \_4$

Vatistas, G. H., Lin, S. \& Kwok, C. K. (1986). Theoretical and experimental studies on vortex chamber flows, AIAA Journal 24(4): 635-642.

URL: $h t t p: / / d x$. doi.org/10.2514/3.9319 
Vyas, A. \& Majdalani, J. (2006). Exact solution of the bidirectional vortex, AIAA Journal 44(10): 2208-2216.

URL: http://dx.doi.org/10.2514/1.14872

Wendt, B. J. (2001). Initial circulation and peak vorticity behavior of vortices shed from airfoil vortex generators, Technical Report NASA/CR-2001-211144, NASA.

$\mathrm{Wu}, \mathrm{J}$. Z. (1986). Conical turbulent swirling vortex with variable eddy viscosity, Proceedings of the Royal Society of London, Series A 403(1825): 235-268.

URL: $h t t p: / / d x$.doi.org/10.1098/rspa.1986.0011

Yan, L., Vatistas, G. H. \& Lin, S. (2000). Experimental studies on turbulence kinetic energy in confined vortex flows, Journal of Thermal Science 9(1): 10-22.

URL: http://dx.doi.org/10.1007/s11630-000-0040-z

Zhang, Z. \& Hugo, R. J. (2006). Stereo particle image velocimetry applied to a vortex pipe flow, Experiments in Fluids 40: 333-346.

URL: $h t t p: / / d x . d o i . o r g / 10.1007 / s 00348-005-0071-z$ 


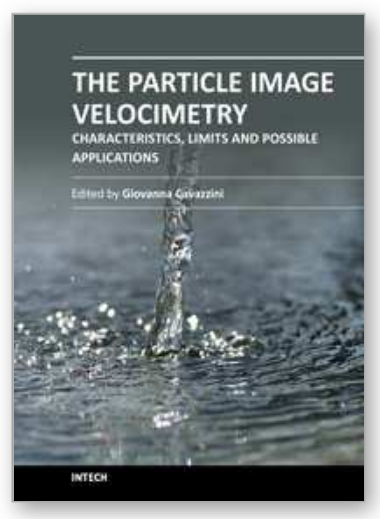

\author{
The Particle Image Velocimetry - Characteristics, Limits and \\ Possible Applications \\ Edited by PhD. Giovanna Cavazzini
}

ISBN 978-953-51-0625-8

Hard cover, 386 pages

Publisher InTech

Published online 23, May, 2012

Published in print edition May, 2012

The Particle Image Velocimetry is undoubtedly one of the most important technique in Fluid-dynamics since it allows to obtain a direct and instantaneous visualization of the flow field in a non-intrusive way. This innovative technique spreads in a wide number of research fields, from aerodynamics to medicine, from biology to turbulence researches, from aerodynamics to combustion processes. The book is aimed at presenting the PIV technique and its wide range of possible applications so as to provide a reference for researchers who intended to exploit this innovative technique in their research fields. Several aspects and possible problems in the analysis of large- and micro-scale turbulent phenomena, two-phase flows and polymer melts, combustion processes and turbo-machinery flow fields, internal waves and river/ocean flows were considered.

\title{
How to reference
}

In order to correctly reference this scholarly work, feel free to copy and paste the following:

Brian A. Maicke and Joseph Majdalani (2012). Characterization of the Bidirectional Vortex Using Particle Image Velocimetry, The Particle Image Velocimetry - Characteristics, Limits and Possible Applications, PhD. Giovanna Cavazzini (Ed.), ISBN: 978-953-51-0625-8, InTech, Available from: http://www.intechopen.com/books/the-particle-image-velocimetry-characteristics-limits-and-possibleapplications/characterization-of-the-bidirectional-vortex-using-particle-image-velocimetry

\section{INTECH}

open science | open minds

\author{
InTech Europe \\ University Campus STeP Ri \\ Slavka Krautzeka 83/A \\ 51000 Rijeka, Croatia \\ Phone: +385 (51) 770447 \\ Fax: +385 (51) 686166 \\ www.intechopen.com
}

\author{
InTech China \\ Unit 405, Office Block, Hotel Equatorial Shanghai \\ No.65, Yan An Road (West), Shanghai, 200040, China \\ 中国上海市延安西路65号上海国际贵都大饭店办公楼405单元 \\ Phone: +86-21-62489820 \\ Fax: +86-21-62489821
}


(C) 2012 The Author(s). Licensee IntechOpen. This is an open access article distributed under the terms of the Creative Commons Attribution 3.0 License, which permits unrestricted use, distribution, and reproduction in any medium, provided the original work is properly cited. 